\title{
¿El retorno de la larga duración?: reflexiones desde Latinoamérica a partir del "History Manifesto of Cambridge"
}

\section{The Return of Long Duration? Reflections from Latin America Revolving Around the "History Manifesto of Cambridge"}

\author{
David Aceituno Silva \\ Instituto de Historia. Pontificia Universidad Católica de Valparaíso (Chile) \\ david.aceituno@pucv.cl \\ Ricardo Iglesias Segura \\ Instituto de Historia. Pontificia Universidad Católica de Valparaíso (Chile) \\ ricardo.iglesias@pucv.cl
}

\section{Resumen}

Los historiadores que impulsan el reciente debate sobre "el Manifiesto de Cambridge sobre la historia" han postulado el surgimiento de una crisis en nuestra disciplina, pero también algunos caminos para resolverla. Las reflexiones que planteamos en este artículo examinan críticamente los postulados del Manifiesto desde una mirada latinoamericana. Se pretende examinar aquellos aspectos que aparecen más débiles, pero también destacar las reflexiones que podrían abrir nuevos análisis sobre la historia y su pensamiento que nos ayude a superar la contingencia o las propias demandas que las humanidades plantean en la actualidad.

\section{Palabras Claves}

Usos de la Historia, cortoplacismo, larga duración, Manifiesto, Latinoamérica

\begin{abstract}
Historians, who have promoted the recent debate on "the History Manifesto of Cambridge", have postulated the emergence of a crisis in our discipline, albeit they have offered ways out to solve too. The reflections we propose in this article are, therefore, addressed to critically analyzing the Manifesto's suggestions from a Latin American standpoint. We shall highlight those aspects that appear to be weakest, but also those reflections that can open up new analysis on history and its thinking, which helps us overcome the contingency or demands Humanities are posing today.
\end{abstract}

\section{Key words}

Uses of History, short-term, Manifesto, long-term, Latin America 


\section{La influencia de Braudel. Historia global y larga duración}

La influencia de Fernand Braudel en las miradas acerca de la Historia y su cambio de paradigma es reconocido mundialmente en todas las escuelas de Historia. Es cierto que su legado es fruto de lecturas de la realidad que se inician con Marc Bloch y Lucien Fevre en los Annales d'histoire économique et sociale, ${ }^{1}$ pero será él quien después de la segunda Guerra mundial consolidará, en especial con la publicación del libro El Mediterráneo y el mundo mediterráneo en la época de Felipe II, una visión acerca de la Historia donde se planteará el estudio que consideró los diversos "tiempos históricos". ${ }^{2}$ Con este trabajo surge una nomenclatura que será utilizada en estudios y reflexiones historiográficas hasta el día de hoy. La "larga duración" será el análisis de las estructuras o aquellos marcos económicos, geográficos e incluso espirituales que perviven y sobrepasan el acontecimiento y se logran distinguir claramente en los periodos largos de tiempo, la "coyuntura" o el tiempo medio serán por ejemplo las variaciones de salarios, de la tasa de interés, etc., y el "tiempo corto" se identificará con el acontecimiento, lo episódico e individual. ${ }^{3}$

Esta forma de entender la temporalidad en la investigación histórica vino a romper la larga tradición de investigación con un fuerte énfasis en lo evenemencial o episódico de la Historia, y puso en la primera línea a los estudios que hacían referencias a lo estructural y coyuntural. Será el propio Braudel que en su lenguaje metafórico expresará la menor consideración que tendrá hacia el estudio de lo individual y episódico al hablar de ella como la "espuma de las olas" de la Historia, aquel tiempo del cual se debe desconfiar, porque dicha "[...] masa no constituye toda la realidad, todo el espesor de la historia, sobre el que la reflexión científica puede trabajar a sus anchas. La ciencia social casi tiene horror del acontecimiento. No sin razón: el tiempo corto es la más caprichosa, la más engañosa de las duraciones". 4

Otro aspecto, no menos relevante del pensamiento "braudeliano", es la noción de Historia "global" o "total", que define Aguirre como "[...] un horizonte de la historia global, que no debe confundirse con la simple e imposible exigencia de una erudición infinita, ni tampoco con la idea igualmente elemental de un ocuparse de todo sin orden ni sentido algunos". En este sentido, la historia total no es una historia general ni mucho menos una universal, en el sentido positivista, donde se buscaría describir cada acontecimiento y suceso acaecido sino que es más bien el intento por mantener la unidad de lo social y la de las distintas realidades sociales e históricas, buscando crear una forma de aproximarse a dichas realidades de manera unitaria y global.

\footnotetext{
${ }^{1}$ Peter Burke, La revolución historiográfica francesa. La escuela de Annales, 1929-1989 (Barcelona: Gedisa, 1993).

${ }^{2}$ Carlos Aguirre, Ensayos braudelianos: Itinerarios intelectuales y aportes historiográficos de Fernand Braudel (Rosario: Argentina, 2000).

${ }^{3}$ Fernand Braudel, El Mediterráneo: el espacio y la historia (México: Fondo de Cultura Económica, 1989); y Fernand Braudel, Escritos sobre la historia (México: Fondo de Cultura Económica, 1991).

${ }^{4}$ Fernand Braudel, La Historia y las Ciencias Sociales (Madrid: Editorial Alianza. S. A., 1970), 64.

${ }^{5}$ Carlos Aguirre, Fernand Braudel y las Ciencias Humanas (Barcelona: Editorial Montesinos, 1996), 35-62.
} 
Sin embargo, lo que resulta más relevante es que gran parte de su pensamiento nace en su estancia en Brasil, quien llega producto de la crisis en Francia a la recién creada Universidad de São Paulo. ${ }^{6}$ Específicamente su obra del Mediterráneo, entre la década del 30 y 40 se nutrirá de su experiencia brasileña, en especial en su comprensión de la larga duración, ya que será en este país donde encontrará nuevas dimensiones geográficas y nuevas características naturales, todo lo cual le sobrecogió sobremanera (hay que recordar que incluye como tesis secundaria una investigación del Siglo XVI brasileño), ${ }^{7}$ al punto que le dio la oportunidad de re-imaginar Europa, en especial ese paisaje tan basto como desconocido que era el mediterráneo. ${ }^{8}$ Evidentemente el influjo de Braudel no se limita a su escrito más famoso, sino que incluye el impacto intelectual que esto generó entre los diversos intelectuales y de manera particular al pensamiento histórico en Brasil. ${ }^{9}$

Será así, como su desarraigo, primero en Argelia y luego en Brasil, terminarían por romper su herencia geográfica, lo que llevará su pensamiento acerca de la historia y las ciencias sociales, un paso más adelante. Como señala Canto, respecto a la experiencia vital de Braudel y su evolución intelectual:

[...] ¿habrán tenido la misma coyuntura los hombres del Mediterráneo que continuamente se veían obligados a entablar viajes prolongados con tal de intercambiar sus productos? La historia del Mar Interior, desde sus inicios más remotos, se haya plagada de intercambios, por lo que la naturaleza misma del hombre mediterráneo es el continuo desplazamiento e interrelación con los demás puntos de la costa mediterránea. ${ }^{10}$

En este sentido, y alejándose del pensamiento de Vidal de La Blache, definió la geohistoria como el estudio de las relaciones económicas, culturales y de intercambio que los hombres entablan -trazando rutas, forjando alianzas- en un espacio geográfico a través de una duración muy larga. ${ }^{11}$

Como se puede ver, no es solo la larga duración, sino que la comprensión compleja de un entramado de relaciones de variado tipo, donde las relaciones entabladas por los hombres afectan claramente los paisajes y sus dinámicas. Su paso por prisión durante la segunda

\footnotetext{
${ }^{6}$ Ian Merkel, "Fernand Braudel, Brazil, and the Empire of French Social Science Newly Translated Sources from the 1930s", French Historical Studies, vol. 40, 1 (2017): 129-160.

${ }^{7}$ Erato Paris, "L'époque brésiliene de Fernand Braudel (1935-1937) et les origines intellectuelles de La Mediterranée et le méditerranéen à l'époque de Philippe II", Storia della Storiografia, 30 (1996): 31-56.

${ }^{8}$ Luís Corrêa Lima, Fernand Braudel e o Brasil. Vivência e Brasilianismo. 1935-1945 (Brasil: EDUSP, 2010).

${ }^{9}$ Paulo Iumatti, "Pontos de partida para o estudo do diálogo entre Caio Prado Jr. e Fernand Braudel”, en Antonio Gilberto Nogueira y Adelaide Gonçalves (eds.), Caio Prado Jr: Legado de um saber fazer histórico (Sao Paulo: Plebeu Gabinete de Leitura, 2013), 49-86; Carlos Guilherme Mota, "Ecos da historiografia francesa no Brasil: Apontamentos e desa-pontamentos", en Leyla Perrone-Moisés, In Do positivismo à desconstrução: Idéias francesas na América (Sao Paulo: EdUSP, 2003).

${ }^{10}$ Emiliano Canto, "Un texto en tres duraciones: Braudel y El Mediterráneo", Temas Antropológicos, Revista Científica de Investigaciones Regionales, vol. 34, 2 (2012): 155-178.

${ }^{11}$ Joël Cornette, “Fernand Braudel et l'économie-monde", L'Histoire, 270 (2002): 51-53.
} 
guerra mundial, también marcará su teoría del tiempo, estableciendo las tres dinámicas: corta, media y larga duración. ${ }^{12}$

\section{¿Cortoplacismo y larga duración?: miradas desde el History Manifesto}

Considerado de esta manera los estudios de la larga duración y su influencia en la historiografía mundial eran bastante promisorios en su desarrollo -y así lo fue durante varios años- pero algo habría ocurrido desde los años setenta del siglo XX en adelante en relación con los estudios históricos y con el propio quehacer del historiador que cambió el rumbo de las investigaciones, o por lo menos, así lo proponen los escritores del "Manifiesto de Cambridge". Debemos señalar que esto no es un descubrimiento en sí mismo, el mismo Braudel de manera autocrítica lamentaba que su tesis no hubiera sido leída fuera de los círculos de historiadores. ${ }^{13}$ Por su parte sus discípulos (Ferro, Duby, Chaunu, etc.), tampoco habían gustado de seguir su mirada de la Historia Global, centrándose en problemas más específicos y diversos, aunque seguían su teoría de la historia en cada investigación. En este sentido Braudel es un caso único de un trabajo de dimensiones notables, pero que vería diluir sus principios en la historiografía posterior, no solo por razones académicas, sino también por otras dinámicas sociales. ${ }^{14}$

Será en este último aspecto donde resulta interesante el trabajo realizado el año 2014 titulado originalmente como "The History Manifesto", que fue escrito por el historiador de las ideas y de Gran Bretaña, David Armitage de la Universidad de Harvard y la profesora de la Universidad de Brown, Jo Guldi. ${ }^{15}$ Según los autores la Historia habría dejado sistemáticamente de interesarse por problemas de larga duración, dejando de lado los postulados más interesantes de Braudel y centrándose en estudios de más corto plazo. El "cortoplacismo" sería según los autores el gran mal de la investigación actual, una especie de epidemia. El texto, de manera evidente buscaba tensionar la historiografía y generar una nueva polémica que hiciera fluir nuevos debates sobre la Historia y el presente, de manera especial en aquellos aspectos que vinculan al pensamiento histórico con los debates políticos contemporáneos. $^{16}$

Sus críticas se centran en dos aspectos fundamentales: el primero, la historia profesional especializada está fuera de la realidad y por tanto lejos de la lectura del gran público y lo segundo, el historiador está desplazado de la toma de decisiones, reemplazado por economistas y cientístas políticos, expertos en analizar la contingencia. Su tesis se afirma en un estudio cuantitativo realizado por Benjamin Schmidt quien señala que desde 1890 en adelante se habría generado en Estados Unidos una contracción de los estudios de largo plazo de las investigaciones doctorales, bajando de un promedio de 70 años hacia fines del siglo

\footnotetext{
${ }^{12}$ Louis-Bernard Robitaille, "El Mediterráneo en los tiempos de Braudel”, Revista Historias, 13 (1986): 3-10.

${ }^{13}$ E. Canto, "Un texto en tres duraciones: Braudel y El Mediterráneo", 173.

${ }^{14}$ François Ewald, "El Imperio de una historia", Revista Historias, 13 (1986): 10-15.

${ }^{15}$ Se puede descargar en: http://historymanifesto.cambridge.org. [consulta 5 junio, 2018].

${ }^{16}$ Deborah Cohen y Peter Mandler, "The History Manifesto: A Critique", American Historical Review, vol. 120, 2 (2015): 530-542; Lynn Hunt, “Does History Need a Reset?”, Annales: Histoire, Sciences Sociales, vol. $70,2(2015)$.
} 
XIX a menos de 30 años hacia $1970 .{ }^{17}$ Esta situación vivida en Estados Unidos, también comenzado a suceder en Europa, debido al aumento de la oferta de programas de doctorados, vinculados normalmente a becas o financiamientos que tienen un tiempo límite de cobertura, todo lo cual habría llevado a muchos doctorandos a buscar temáticas más abordables, considerando el tiempo de investigación que tenían disponible acortando por tanto sus investigaciones.

De esta manera, señalan:

\begin{abstract}
A medida que los historiadores del "corto plazo" comenzaron a repensar su relación con los archivos y las audiencias [...] Con algunas excepciones, las obras clásicas de los años 70, 80 y 90 se concentraron en un episodio particular: la identificación de un trastorno particular dentro de la psicología o el análisis de un motín particular en el movimiento obrero, por ejemplo. Casi todos los historiadores sociales experimentaron en cierto sentido con escritos históricos de corta duración para interactuar [...] cada una de ellas completando un solo episodio en la larga historia del trabajo, la medicina, el género o la domesticidad. ${ }^{18}$
\end{abstract}

Los resultados de esta transformación en el desarrollo de las investigaciones históricas, señalan los autores del Manifiesto, habrían sido también el resultado de otros procesos que se han ido profundizando hasta la actualidad, como son la profesionalización del trabajo del historiador y su vínculo con las demandas de la Universidad contemporánea. En este sentido, la primera batalla que daría la investigación histórica y de las humanidades es la de pensar sus investigaciones mirando más allá de las preocupaciones cortoplacista vinculadas a la financiación, la demanda de acreditación o el cumplimiento de indicadores de productividad que ponen al pensamiento histórico entre la espada y la pared. ${ }^{19}$ Pensar históricamente estaría hoy vinculado a la necesidad de responder a la inmediatez "académica." Una realidad de la cual no solo forma parte Estados Unidos y Europa, sino que está cada vez más presente en América Latina. ${ }^{20}$

Enfrentado a esta realidad, señalan los autores, el historiador se vio envuelto en otro dilema, que se refiere a la perdida de las virtudes distintivas de las humanidades y las ciencias sociales e históricas dentro de ella, en el sentido que las investigaciones de largo plazo tienen el potencial de entregar reflexiones profundas de la relación entre el pasado y el futuro por lo que puede ocupar un lugar especial en la decisión política. Así dicho, la historia -como disciplina y su objeto- enfrentada con el cortoplacismo y el academicismo necesitaría volver a tener un lugar en el debate público en tiempos de crisis. Esto porque la sociedad requiere con urgencia de un pensamiento que supere la contingencia y logre proyectar su análisis sobre el presente más allá de sí mismo, por lo que la Historia estaría llamada a reencontrarse con los "grandes problemas".

\footnotetext{
${ }^{17}$ David Armitage y Jo Guldi, The History Manifesto (Cambridge: Cambridge University Press, 2014 ), 44.

18 Ibid., 45.

${ }^{19}$ João M Paraskeva, Capitalismo académico (Portugal: Pedago, 2009).

${ }^{20}$ Antônio A. S. Zuin y Lucídio Bianchetti, “O produtivismo na era do 'publique, apareça ou pereça': um equilíbrio difícil e necessário”, Cadernos de Pesquisa, vol. 45, 158 (2015): 726-750.
} 
Ahora bien, frente a estos problemas los historiadores del "Manifiesto" recetan un retorno a la larga duración de Braudel, definitivamente no, ya que según ellos, la larga duración y las demandas del presente son muy distintas a las de mediados del siglo XX, tanto en sus temáticas como en sus metodologías. Citan por ejemplo, trabajos que desde perspectivas incluso más amplias que las propuestas del largo tiempo braudeliano, comienzan a abrir caminos nuevos para la investigación histórica como es el caso de la "deep history" 21 y la "big history", ${ }^{22}$ que incluyen periodos de centenas o miles de años, lo cual no significa sólo una ampliación temporal sino que también una ampliación temática, de datos y problemas -vinculados al presente, pero en perspectiva histórica, como son por ejemplo el cambio climático, la gobernabilidad y la desigualdad-.

En este sentido, la "nueva larga duración" es novedosa en sus métodos (el uso de la Historia digital) como por sus temas, que involucran nuevas miradas de interconexión global mediados por las redes sociales y las nuevas tecnologías de los mass media, a su vez que poseerían un dinamismo y flexibilidad impensables para estudios más antiguos. Por ejemplo, se contaría con una nueva reserva de fuentes que ahora son mucho más abundantes, con grandes datos disponibles y bajo novedosas posibilidades de análisis digital. Debido a este mismo aumento de material disponible para la investigación, esta "nueva larga duración" tendría un mayor potencial para los historiadores, los científicos sociales e incluso para el mundo político y el público general. Así sintetiza Julián Casanova las nuevas demandas a las que un historiador se ve enfrentado en la actualidad:

\begin{abstract}
Los historiadores necesitamos años, décadas, para averiguar los hechos más relevantes del pasado, reconstruir el curso de los acontecimientos, sacar a la luz las intenciones de sus protagonistas. Una mirada rigurosa a la Historia exige para nosotros, necesariamente, la aplicación de métodos críticos para evaluar las fuentes, la adopción de técnicas reconocidas para presentar y editar el material y un notable ingenio para detectar los errores en la transmisión de la información y determinar la fiabilidad de los testimonios individuales. Y, de repente, en apenas unos días, la filtración de una masa ingente de material diplomático, obtenida por Wikileaks y divulgada por algunos de los más prestigiosos medios de comunicación internacionales, proporciona una minuciosa crónica de la relación de Estados Unidos de América, el país más poderoso de la Tierra, con el resto del planeta [...] La historia se acelera y ya no podemos aspirar a comprender las cosas pasado el tiempo, con el obligado reposo de las fuentes en los archivos, con el uso de métodos críticos para interpretar los textos y la información. ${ }^{23}$
\end{abstract}

En este sentido, el "Manifiesto" no solo reconoce las deficiencias de la historiografía actual, sino que además pone urgencia a la necesidad de repensarla de una manera distinta, frente a la creciente cantidad de información que las nuevas tecnologías ponen delante del investigador. La Historia en este sentido serviría también como una guía para la acción en el presente, mediante el uso de los recursos del pasado con el fin de imaginar alternativas

\footnotetext{
${ }^{21}$ Andrew Shryock, y Daniel Lord Smail, Deep History: The Architecture of Past and Present (USA: University California Press, 2012).

${ }^{22}$ David Christian, Maps of Time: An Introduction to Big History (USA: University California Press, 2005).

23 Julián Casanova, "Secretos de Estado", El País, 03 de diciembre del 2010. Recuperado de: http://www.elpais.com/articulo/opinion/Secretos/Estado/elpepuopi/20101203elpepiopi 5/Tes [consulta 3 de octubre, 2018].
} 
posibles en el futuro. Estas últimas nacen de la búsqueda de otras concepciones acerca del hombre, que rompen con la parálisis que puede generar el cortoplacismo, sugiriendo nuevas formas de pensar en lo contra-factual, el imaginar utopías y un sentido de destino.

Es interesante hacer notar, que pese al fuerte impacto que ha tenido la historiografía "braudeliana" en Latinoamérica, y la creciente demanda impuesta por los cambios en la Universidad sobre el historiador profesional latinoamericano, el que estas reflexiones sean como conocidas sigue pareciendo singular. ¿Es que quizás acá la larga duración no ha perdido relevancia?, o ¿el historiador sigue siendo influyente en el espacio público?, o ¿simplemente es debido a que el debate surgido en Norteamérica y Europa no es replicable en Latinoamérica? En las líneas siguientes buscamos algunas respuestas a este debate con algunas reflexiones generales que sirvan para iniciar una discusión para nuestro contexto.

\section{La mirada Latinoamericana del Manifiesto}

En el mismo momento en que surgía el debate histórico producto del Manifiesto de Cambridge, se debatía acerca de El Capital del Siglo XXI, de Piketty, ${ }^{24}$ de hecho es incluido por los autores como ejemplo de trabajo de larga duración y del poder de los datos seriados al señalar que:

[...] Piketty explica en su introducción que su motivación para recopilar datos de larga duración sobre la desigualdad se inició cuando escucho la declaración que la mayoría de los economistas aceptan como ley: la afirmación de Kuznets, de que el capitalismo, con el tiempo, tenderá a reducir la desigualdad. ${ }^{25}$

De esta manera, una investigación de larga duración tendría el potencial de cambiar y romper con conocimientos arraigados por décadas y derrumbar tesis consolidadas, como había sido la de la relación capitalismo/desigualdad, al menos para el caso europeo. Resulta razonable pensar que trasladar estas miradas al caso latinoamericano puede suponer un avance en la historiografía, sin embargo no siempre es posible trasladar conceptos y teorías de manera clara al contexto americano. Por ejemplo, si tomamos el mismo caso propuesto por Piketty, vemos que tiene bastantes limitaciones para la situación de Latinoamérica, las cuales han sido reconocidas por el mismo autor. Así, cuando establece la relación $r>g$ (formula que señala que cuando la tasa de retorno sobre el patrimonio [r] es mayor que la tasa de crecimiento $[\mathrm{g}]$, se acelera la concentración de la riqueza) no considera necesariamente otros factores que influyen en gran medida en este lado del mundo, como son por ejemplo el acceso a la educación, el que la acumulación de riqueza no siempre ha sido producto de la herencia o posesión de la tierra (muchos ricos lo son gracias a los beneficios de recientes dictaduras, por ejemplo) ${ }^{26}$ o la intensidad de la acumulación de la

\footnotetext{
${ }^{24}$ Thomas Piketty, El capital en el siglo XXI (México: Fondo de cultura económica, 2014).

${ }^{25}$ D. Armitage y J. Guldi, The History Manifesto, 79.

${ }^{26}$ Véase: María Olivia Mönckeberg, El saqueo de los grupos económicos al Estado de Chile (Chile: Ediciones B, 2001); Serpaj, La complicidad del poder económico con la dictadura: El caso Mercedes Benz (Buenos Aires: Servicio Paz y Justicia Equipo, 2003); Horacio Verbitsky y Juan Pablo Bohoslawsky, Cuentas Pendientes. Los cómplices económicos de la dictadura (Buenos Aires: Siglo XXI Editores, 2013).
} 
riqueza. Esto no quiere decir que sus principios no puedan ser aplicados en Latinoamérica, sino que deben ser atendidos de manera diferente.

En general, el "Manifiesto" tiene la misma limitación que en otro momento lo tuvieron diversas teorías foráneas al contexto latinoamericano, ya que si bien sus conclusiones pueden ser interesantes y pertinentes -en especial en lo que guarda relación con los vínculos entre historia, poder y demandas actuales de la universidad- existen otros aspectos que deben ser matizados o discutidos. El caso de Latinoamérica es complejo, ya que como señala Aguirre no existe aún:

[...] una historia global de la historiografía de América Latina en el siglo XX. Y si no existe esta más particular historia de nuestra historiografía en la última centuria, tampoco existen, más en general, ni una historia intelectual de Latinoamérica en el siglo XX, ni aún una satisfactoria historia general de nuestro semi-continente dentro de este breve siglo XX que corre desde la primera guerra mundial y la revolución rusa, hasta la caída del Muro de Berlín en 1989. ${ }^{27}$

Lo que propone Aguirre es un importante desafío, ya que la historiografía en América Latina tiene como eje articulador el Estado nacional, su formación y desarrollo donde prevalecen las historias nacionales, por sobre las historias regionales o globales como lo propuso Braudel para el caso europeo, donde el mediterráneo era el espacio articulador. Evidentemente ha habido intentos por desarrollar investigaciones regionales, ${ }^{28}$ pero no siempre logran generar lecturas integradas, sino más bien estudios comparativos. ${ }^{29}$ Desde un punto de vista temporal, también se siguen utilizando cronologías divisorias, generando miradas compartimentadas de la historia como sucede por ejemplo con la América precolombina, Colonial y los procesos de independencia.

Por otra parte, durante los últimos años se ha intensificado la investigación de los sucesos más recientes con especial énfasis en los periodos traumáticos de la historia de Latinoamérica, como por ejemplo la Memoria histórica, la Dictadura o los procesos de

\footnotetext{
${ }^{27}$ Carlos Aguirre, "El largo siglo XX de la historiografía latinoamericana contemporánea: 1870-¿2025? Puntos de partida para su reconstrucción”, Historia Agenda, Año I. Nueva Época. 3 (2003).

${ }^{28}$ Podemos destacar los trabajos de Eduardo Cavieres sobre Perú y Bolivia y los de Brasil de Ana María Stuven y Marco Pamplona. Véase: Eduardo Cavieres y Cristoval Aljovín de Losada (comps.), Chile-Perú. Perú-Chile. 1820-1920. Desarrollos políticos, económicos y culturales (Valparaíso: Pontificia Universidad Católica de Valparaíso, Convenio Andrés Bello, Universidad Mayor San Marcos, 2005); Eduardo Cavieres y Fernando Cajías de la Vega (coord.) Chile-Bolivia, Bolivia-Chile: 1820-1930. Desarrollos Políticos, Económicos y Culturales (Valparaíso: Ediciones Universitarias de Valparaíso, 2008); Ana María Stuven y Marco Pamplona (eds.), Estado y Nación en Chile y Brasil en el siglo XIX (Santiago: Ediciones Universidad Católica de Chile, 2009).

${ }^{29}$ John D. French, Magnus Mörner y Julia Fawaz Viñuela, "Comparative Approaches to Latin American History”, Latin American Research Review, vol. 17, 2 (1982): 55-89.
} 
democratización. ${ }^{30}$ Sin embrago, salvo pocos casos sigue persistiendo la mirada nacional. ${ }^{31}$ En este sentido, es menester recoger la crítica hecha por el "Manifiesto", en el sentido que si bien la historia de los diversos países de Latinoamérica han sido estudiados de manera específica, estos siguen estando dominados por la temporalidad decimonónica y la construcción de espacios divisorios basados en imaginarios nacionales que impiden establecer conexiones globales de análisis de largo tiempo. La superación de estas miradas no es fácil, en especial si observamos la historiografía tradicional producida hasta ahora, por lo cual la interdisciplinariedad y las nuevas fuentes de información son relevantes. Sobre esto último hay que señalar que existen dificultades, que ya denotó el mismo Piketty, al encontrarse con carencia de datos seriados o sistematizados lo que hace más difícil la tarea planteada por los historiadores que proponen el "Manifiesto", incluso si se utiliza nueva tecnología en su procesamiento.

Con todo, la larga duración y la mirada global de Latinoamérica sigue siendo una necesidad y un desafío para el historiador. Para esto es necesario superar varios escollos, el primero plantear nuevas temporalidades y lecturas del espacio que nos hagan superar la historiografía nacional.

\section{Repensar el estudio de América Latina en la larga duración: Historia comparada, transnacional y global}

El debate sobre como investigar en Latinoamérica y el esfuerzo por superar los espacios nacionales, no es un problema reciente, lo que hace el "Manifiesto" es sólo apuntar sobre un aspecto que aún no encuentra solución satisfactoria en Europa y Estados Unidos, pero podemos decir que por extensión al espacio americano. Sobre esto, las respuestas que se han buscado van desde las tradicionales historias comparadas, hasta las más nuevas historias conectadas y transnacionales.

Hace bastantes años señalaba O’Gorman la necesidad de mirar la "región” de América para intentar buscar explicaciones históricas de sus procesos y desarrollos, ${ }^{32}$ comparando entre los espacios vecinos, evitando concentrarse demasiado en la contrastación con los procesos europeos, como había sido tradicionalmente propuesto, al intentar buscar los conceptos claves que se podían importar de manera más o menos certera. Los primeros intentos de Historia comparada se remontan a la primera mitad del siglo XX de la mano del historiador mexicano Silvio Zavala, que se pronunciaba sobre los procesos de conquista. ${ }^{33}$ Tanto este como los que se desarrollan posteriormente (al menos hasta los años 70), buscan

\footnotetext{
${ }^{30}$ Véase: Elizabeth Jelin (comp.), Las conmemoraciones: las disputas en las fechas 'in-felices' (Madrid: Siglo XXI, 1998); Martha Herrera y Carol Pertuz, "Educación y políticas de la memoria sobre la historia reciente de América Latina", Revista Colombiana de Educación, 71 (2016): 79-108; Collins Cath, Katherine Hite y Alfredo Joignant, Las políticas de la memoria en Chile: desde Pinochet a Bachelet (Santiago: Ediciones UDP, 2013).

${ }^{31}$ Véase Patrice Mcsherr, Los estados depredadores: la Operación Cóndor y la guerra encubierta en América Latina (Chile: LOM, 2009); Daniel Camacho y Rafael Menjívar, Los Movimientos populares en América Latina (Chile: Siglo XXI editores, 2005).

32 Edmundo O’Gorman, La invención de América (México: Fondo de Cultura Económica, 1977).

${ }^{33}$ Silvio Zavala, Las conquistas de Canarias y América (Canaria: Cabildo Insular de Gran Canaria 1991).
} 
dar cuenta de ciertas "causas generales de los fenómenos históricos, que pretende llegar a generalizaciones y que va en dirección a la construcción de modelos", como señala Coelho. ${ }^{34}$ Esta perspectiva, como queda claro, tenía una visión macro-histórica, donde los problemas comunes eran muy relevantes de estudiar, especialmente porque se necesitaba entender el presente, especialmente en aquello que ayudaba a comprender el subdesarrollo de la región. Hay que destacar que desde que se inicia la Historia comparada en América, en Europa se vive una tensión interesante, especialmente con los historiadores franceses, como era el caso de Marc Bloch, quien proponía una historia comparada, donde Francia y su momento revolucionario podría ser un eje para comprender occidente. ${ }^{35}$ Por su parte, la Ciencia política argumentaba a favor de análisis que ponían en el centro a Estados Unidos, en referencia por ejemplo al desarrollo de la democracia en relación con el liberalismo. ${ }^{36}$

De manera más reciente, han aparecido nuevas interpretaciones para entender los problemas regionales. Una de estas miradas es la de las Historia Conectadas propuesta por el investigador indio Sanjay Subrahmanyam, que busca alejarse de la explicación eurocéntrica de los problemas de moderna de Eurasia. ${ }^{37}$ Esta teoría es recogida en América Latina por algunos investigadores para criticar la historia comparada señalando que refuerza la visión eurocéntrica, en especial respecto a sus miradas dualistas del mundo. La Historia conectada es la de la complejidad, por ejemplo la que pone de manifiesto el entrecruce cultural y el mestizaje. ${ }^{38}$

Otra mirada que ha buscado la superación de los estados nacionales en la investigación histórica, es la que se denomina Historia transnacional, en este caso su mirada está puesta en destacar las interconexiones de la historia de la humanidad pensada sin fronteras, donde se puede enfatizar las redes, los procesos, las creencias y las instituciones, trascendiendo el espacio nacional, para lo cual se puede utilizar cualquier método y problema (económico, social, cultural, etc.). Lo principal en esta mirada historiográfica tiene que ver con la comprensión de las circulaciones y los movimientos. ${ }^{39}$ Esta mirada a veces se confunde con la de la Historia Global o el "giro global", que es otra línea de investigación actual donde busca construir narrativas de vida global, pero donde se busca entender la integración como la desintegración. ${ }^{40}$

\footnotetext{
${ }^{34}$ María Ligia Coelho, "América Latina: historia comparada, historias conectadas, historia transnacional", Anuario Facultad de Humanidades y Artes (UNR), 24 (2011): 11.

${ }^{35}$ Marc Bloch, "Pour une Histoire Comparée des sociétés européennes", Mélanges historiques, 1 (1963).

${ }^{36}$ David M. Ricci, The Tragedy of Political Science. Politics, Scholarship and Democracy (Londres: Yale University Press, 1984).

${ }^{37}$ Sanjay Subrahmanyam, "Connected histories: notes towards a reconfiguration of early modern Eurasia", Víctor Lieberman (ed.), Beyond Binary Histories. Re-imagining Eurasia to c. 1830 (Michigan: The University of Michigan Press, 1999).

${ }^{38}$ Serge Gruzinski, O pensamento mestiço (Sao Paulo: Companhia das Letras, 2001).

${ }^{39}$ Isabel Hofmeyr y Matthew Connelly, "AHR Conversation: On Transnational History”, American Historical Review, vol. 111, 5 (2006): 1441-1464.

${ }^{40}$ Bruce Mazlish, "Comparing Global History to World History", The Journal of Interdisciplinary, vol. 28, 3 (1998): 385-395.
} 
Como podemos ver, si bien el "Manifiesto" hace un acento sobre la necesidad de repensar el largo plazo y "descentrar" la mirada poniendo énfasis en problemas más complejos y alejados del Estado Nacional, esto se viene intentando en América latina desde hace varias décadas, incluso con una importante producción en los años 60 de la mano de los teóricos de la dependencia. Sin embargo, resulta evidente que se hace necesario seguir analizando los resultados de estas nuevas miradas teóricas sobre lo transnacional y lo global, ya que aún siguen estando muy vigentes las miradas menos integradas de los problemas Latinoamericanos. $^{41}$

\section{América Latina y los estudios del presente extendido.}

Si, como hemos visto, la discusión sobre miradas más integradoras denota un avance interesante en América Latina, también lo encontramos en lo referido a los problemas del tiempo largo, que incorporando el presente va más allá de él. Es lo que sucede con las investigaciones de los Historiadores del "tiempo presente", quienes incorporan el concepto de tiempo extendido en sus análisis. Estos investigadores proponen que no es lo mismo hacer estudios cortoplacistas que estudios de tiempo corto. Los primeros se refieren más bien a la coyuntura por la coyuntura o en otras palabras, a investigaciones que solo se enfocan en aspectos tan específicos que no permiten hacer generalizaciones o alguna reflexión útil para la sociedad. Sin embargo, desde los años setenta se vienen desarrollando investigaciones del "tiempo presente" o de "historia actual", que se enfocan en análisis de temáticas muy recientes (el lapso de una vida o una generación) que son capaces de apropiarse de realidades profundas enraizadas. ${ }^{42}$

Este tiempo extendido, como señalamos, es para el historiador del tiempo presente el lugar de una temporalidad extendida que contiene la memoria de las cosas pasadas experiencia- y la expectativa de las cosas por venir. ${ }^{43}$ Un lugar de transición entre lo que fue futuro y lo que deviene del pasado, una especie de continuum, como señala Koselleck:

[...] Desde un punto de vista puramente teórico, toda historia puede ser definida como un presente permanente en el cual están contenidos el pasado y el futuro, o, también, como el entrecruzamiento constante del pasado y el futuro. Esto provocaría la desaparición de todo presente. En el primer caso, en que se hace hincapié sobre la sincronía, están contenidas simultáneamente todas las dimensiones temporales. En el segundo caso, en que se destaca la diacronía, la presencia activa de los hombres no tiene, históricamente hablando, ningún campo de acción. ${ }^{44}$

\footnotetext{
${ }^{41}$ Vease: Barbara Weinstein, "Pensando la historia más allá de la nación: la historiografía de América Latina y la perspectiva transnacional", Aletheia, vol. 3, 6 (2013).

${ }^{42}$ Francois Bédarida, "Definición, método y práctica de la Historia del Tiempo Presente", Cuadernos de Historia Contemporánea, 20 (1998).

${ }^{43}$ Reinhart Koselleck, Futuro pasado. Para una semántica de los tiempos histórico (Barcelona: Paidós, 1993).

${ }^{44}$ Reinhart Koselleck, "Einleitung", Geschichtliche Grundbegriffe, XXVII. Citado en Alejandro Cheirif, "La teoría y metodología de la historia conceptual en Reinhart Koselleck", Historiografías, Revista de historia y teoría, 7 (2014): 92.
} 
De esta manera, se hace posible que el historiador pueda estudiar el presente y al hacerlo no deja el pasado y sus vestigios ni tampoco se desentiende del presente, ya que estos momentos confluyen en este presente dilatado. Este tipo de investigaciones es particularmente interesante para los espacios e historias como la latinoamericana que está llena de episodios violentos o traumáticos que marcan ciertas temporalidades haciéndolas permanentes en el tiempo y que permitirían unirlas para, entenderlas en el largo o mediano tiempo aun siendo estudios del presente (memoria y violencia, por ejemplo). Como señala Mateos:

[...] los criterios de periodización de la Historia del presente han partido habitualmente de acontecimientos nodales, de hechos fundadores ligados a una gran convulsión política (una guerra, una revolución) que afecta de manera decisiva a una determinada historia nacional. No deja de resultar significativo que la noción de tiempo presente se esté desarrollando en países de Europa continental o de Iberoamérica afectados por acontecimientos que aparentemente significaron fuertes rupturas históricas. ${ }^{45}$

Una interesante solución para los estudios de largo tiempo en Latinoamérica podría ser, paradojalmente, desarrollar investigaciones del tiempo presente, entendiendo que este no sólo estudia lo que acontece hoy día sino que busca las conexiones en ese tiempo extendido, a veces perdido en el pasado y que pervive a veces sin percatarnos hasta el propio presente. ${ }^{46}$ Para el caso chileno, existen diversos ejemplos de este tipo de estudio que han aportado con nuevas miradas la Historia política, como es el caso de Ángel Soto, Isabel Torres, o Pablo Rubio Apiolaza, ${ }^{47}$ a su vez se ha creado una Red interdisciplinaria de estudios de la historia reciente (RIEHR) ${ }^{48}$ donde participan investigadores chilenos, argentinos, españoles y de otras partes del mundo, la Asociación de historia actual, la revista HAOL (Historia actual on line) e Historia a debate que reúnen en su web investigaciones de todo el mundo y que han servido como plataforma de debate de historiadores chilenos que se han ido acercando a esta línea historiográfica.

El género sobre el cual se ha realizado trabajos en relación a la historia y la memoria ${ }^{49}$ son amplísimos: memoria familiar (memorias intimistas, afectivas, ritualistas, socioeconómicas, etc.), memoria histórica (en relación con una clase, pueblos, nación y con sus acepciones de memoria obrera, popular, etc.), memoria/olvido y los lugares de la memoria. Justamente es en este último tópico es donde se ha avanzado bastante en su relación con la historia del tiempo presente, ya que en este caso la cronología y la relación

\footnotetext{
45 Abdón Mateos, "Historia, Memoria, Tiempo Presente", Hispania Nova, 1 (1998).

46 Véase: David Aceituno, "Historia del tiempo presente: reflexiones para la investigación en Chile”, en David Aceituno y Baldomero Estrada (coords.), Reflexiones y Miradas a nuestro Pasado Reciente. Actas de la I Jornada de Historia de Chile Contemporáneo, Instituto de Historia (Valparaíso: PUCV, 2013), 23-39.

${ }^{47}$ Isabel Torres, La crisis del sistema democrático (Santiago: Ed. Universitaria. 2014); Pablo Rubio, Los civiles de Pinochet. La derecha en el régimen militar chileno, 1983-1990 (Santiago: Dibam-Centro de Investigaciones Diego Barros Arana, Santiago, 2013); Ángel Soto, "Historia del presente: estado de la cuestión y conceptualización”, Historia Actual Online, HAOL 3 (2004): 101-116.

48 Véase: http://www.riehr.com.ar [consulta 27 de junio, 2019].

${ }^{49}$ Paul Connerton, How Societes Remember (Cambridge: University Press, 1989).
} 
pasado/presente generan un buen espacio de investigación para aquellos problemas donde es menos importante el análisis de los hechos y su memorización. ${ }^{50}$

Para historiadores del presente, esto significa a su vez una revitalización de la Historia política, lo que a su vez es la respuesta a las demandas por más historia desde una mirada de la Historia de los conceptos, que puede nutrir la Historia del presente en América Latina. ${ }^{51}$ Como señala Rosanvallon:

[...] el estudio del lenguaje político desde la historia política comenzó a dar un giro trascendental. La política pasó a ser comprendida como una lucha por los sentidos, por refundar o recrear nuevas matrices categoriales, luchas por la significación de la realidad y por ende, luchas por redefinir los espacios y constituir las conductas más apropiadas para alcanzar un determinado proyecto. ${ }^{52}$

Hay que hacer énfasis que la mirada del historiador del presente y sus estudios, distan del presentismo. De hecho autores señalan justamente que uno de los objetivos que se tiene al desarrollar investigaciones que consideren una nueva teoría histórica del tiempo presente, es enfrentar el mal del presentismo que "exhibe la problemática de pérdida de sentido histórico" donde "[...] la fuerza del presentismo es empujar a los sujetos a un tipo de "condición histórica donde no están afectados por el pasado ni el futuro", como señala Ovalle. ${ }^{53}$

\section{Por un nuevo historiador para el S. XXI}

Además de las reflexiones acerca de qué historia necesitamos, los autores del "Manifiesto" plantean preguntas muy relevantes para el historiador del siglo XXI que nos confronta con nuestra propia profesión. ¿Qué hemos perdido? ¿Qué rol debemos cumplir? Sus análisis parten haciendo notar que existe una verdadera crisis en la sociedad debido a que toda actividad esta reducida a su mínima expresión, donde la inmediatez es lo dominante y en la que casi no existen proyectos de larga datación. De esta manera, los políticos se encuentran en permanente campaña y por lo tanto los resultados son lo más determinante, sobre todo si se piensa en el siguiente mandato. Todo se planifica en corto plazo, porque los recursos están sometidos a la evaluación constante, por lo que nadie se atreve a hacer proyecciones de largo aliento, un nuevo trabajo - precario muchas veces- una ONG, o la

\footnotetext{
${ }^{50}$ Por ejemplo: Fernando Camacho, "Memorias enfrentadas: las reacciones a los informes nunca más de Argentina y Chile”, Persona y sociedad, 67, vol. XXII, 2 (2008): 67-99; Brian Loveman y Elizabeth Lira, Políticas de reparación. Chile 1990-2004 (Santiago: LOM Ediciones, 2005); Nelly Richard, Latencias y sobresaltos de la memoria inconclusa (Chile 1990-2015) (Córdoba: Editorial Villa María, 2018).

${ }^{51}$ Cristina Moyano, "La historia política en el bicentenario: entre la historia del presente y la historia conceptual. Reflexiones sobre la nueva historia política”, Revista de Historia Social y de las Mentalidades, vol. 15, 1 (2011): 227-245.

52 Pierre Rosanvallon, "Para una historia conceptual de lo político. Nota de trabajo", Prismas Revista de Historia Intelectual, 6 (2002): 123-136.

${ }^{53}$ Daniel Ovalle, "Pensar la conciencia histórica contemporánea. Historicidad y teoría de la historia", en Pablo Aravena (ed.), Representación histórica y nueva experiencia del tiempo (Valparaíso: América en movimiento, 2019), 49-63.
} 
administración estatal, todos están en una situación que denominan como de un constante "calentando motores" con una atención patológica por el corto tiempo. ${ }^{54}$

En este círculo vicioso de demandas constantes y apremio por el tiempo, se perdieron los mitos y proyectos de larga duración. A su vez, los cientistas sociales han optado por el acercamiento teórico matemático a la realidad de manera que muchos de los acontecimientos humanos se han reducido a modelos derivados de la física, traducido por economistas. La televisión y los noticieros recogen estas lecciones y las repiten todos los días educando a la gente, con lo cual la sociedad se ha reducido a explicaciones basadas en modelos matemáticos o en base a hechos aislados unos de otros, a lo sumo, un anecdotario histórico. Pese a esto la gente sigue haciendo grandes preguntas que no son posibles de contestar bajo estos reduccionismos, por ejemplo, qué alternativas existen para el neoliberalismo, cómo abordar la migración, la desigualdad, la sostenibilidad, etc., todas ellas inquietudes que solo una disciplina humana compleja y abierta a la reflexión con otras humanidades puede responder, aquella llamada a analizar los cambios y las permanencias, como es la Historia.

Para el caso Latinoamericano, es cierto que ha habido y hay historiadores que participan de discusiones públicas, e incluso de la toma de decisiones. A su vez, al menos para el caso chileno, durante los últimos años se han escrito varios libros de difusión histórica que se han transformado en Best Sellers (aunque no fueron escritos por un historiador profesional) ${ }^{55}$ y se ven como nunca antes comentarios "históricos" en televisión. A la par que esto sucede, en Chile se comienza a disminuir las horas de Historia en las escuelas. Esta paradoja es entendible cuando se conoce qué la historia que se difunde, es una historia light de consumo general, ya que lo que el público consume son las "anécdotas" históricas, las historias secretas, conspiraciones, etc., y no necesariamente las grandes explicaciones o los complejos problemas de nuestra región y país. En la práctica la Historia reflexiva y crítica sigue estando lejos del público general.

Por esto, vale la pena seguir preguntándose: ¿Quién está escribiendo hoy día acerca de las épocas y siglos que dan sentido al presente? ¿Quién prepara investigaciones globales de largo alcance que pongan en evidencia las continuidades de la Historia? Es difícil señalarlo, pensando en las demandas a la que se ve sometido el historiador profesional, porque esta misma necesidad de conseguir recursos para seguir investigando produce que sus escritos se alejen del público general y sus problemas, generando una mayor brecha entre la Historia académica y su percepción de utilidad. Ya lo enunciaba García de Cortázar, catedrático español cuando señalaba que:

[...] aquellos ilusos que aspiraban a estudiar el "paso de una estructura a otra" y explicar sus errores, temer sus equivocaciones, y prometer sus correcciones, han sido arrojados a las tinieblas de los campus, al polvo de los legajos, al revistero minúsculo y secreto. Sus hábiles sustitutos, maestros en el uso de la retórica leguleya y en dominar situaciones imprevistas, no

\footnotetext{
${ }^{54}$ D. Armitage y J. Guldi, The History Manifesto, 2.

${ }^{55}$ Jorge Baradit, Historia secreta de Chile (Santiago: Editorial Sudamericana, 2015); Id., Historia secreta de Chile II (Santiago: Editorial Sudamericana, 2016); e Id., Historia secreta de Chile III (Santiago: Editorial Sudamericana, 2017).
} 
quieren complicaciones administrativas y les buscan dorados exilios internos, en la confianza de que son una especie a extinguir. El tiempo que tanto aprecian se encargará de ellos.

Mientras tanto, las cosas se van haciendo sin su concurso (y sin el de filósofos y poetas) cada vez más parcelarias, funcionales y deshumanizadas. "Nadie que sepa historia entre aquí", parecen parodiar los frontispicios de los parlamentos, los gobiernos, los ministerios... Es decir, nadie que crea que puede decirnos en qué nos equivocamos y como debemos llevar las cosas. A ser posible que tampoco el pueblo ("eso" que nos vota cada cierto tiempo) sepa historia. Cuatro reglas, tres idiomas y algo de tecnología es suficiente para el sentido común postmoderno.

Y la clase política, aislada del pensar histórico, lejos de la confrontación con la realidad (que pedía Dewey), preocupada en su "minusculidad", desentendida de todo lo que no la haga crecer un centímetro o engordar un gramo, arroja por la ventana los hechos, su significado y sus consecuencias. $^{56}$

Pero la culpa de esto, como hemos visto, no es solo del que quiere dejar fuera a la Historia de la reflexión pública, sino que también es del historiador quien ha decidido someterse al pensamiento cortoplacista, entendible en muchos casos debido a la necesidad que imponen los procesos de acreditación y financiamiento. Pese a todo, como hemos visto existen intentos de diverso tipo para superar esta encrucijada, con miradas integradas, para pensar el presente y con cada vez mayor difusión.

\section{Reflexiones finales}

Las propuestas del "Manifiesto" son de sobremanera críticos para la disciplina histórica, siendo un llamado de alerta a repensar sus objetivos, en especial pensando en el contexto norteamericano y europeo. Sin embargo, consideramos que para el caso latinoamericano podemos ser optimistas, en el sentido, que la Historia y las Ciencias sociales llevan varios años buscando caminos para dar miradas globales a la región. Por una parte creemos que pese a las críticas que pueden surgir del trabajo del historiador como académico, es la Universidad el espacio principal para pensar la sociedad a largo plazo, independientemente que hoy se vea sometidas a los avatares presupuestarios y las requeridas acreditaciones. Es justamente el espacio institucional universitario donde se encuentra la libertad para enfrentar los desafíos del presente, sin la tensión del espacio público, pero pensando influir en él. Resulta evidente que la política, los problemas ambientales y sociales, etc., y principalmente una comprensión más clara de lo que sucede en América Latina de manera integrada se hace necesario, sobre todo si se piensa en la falta de información respecto al ascenso de los extremos en la política y nuestro propio pasado cercano, que nos habla de la fragilidad democrática que vivimos.

Por otra parte, abogamos por la Historia como una disciplina humana crítica y que busque instalar nuevas formas de entender el presente en el largo plazo, en especial en una Latinoamérica que vinculada a sus historias nacionales tiende a dividir más que a unir.

\footnotetext{
${ }^{56}$ Fernando García de Cortázar, "El historiador entre el poder y la nada", Cuenta y razón del pensamiento actual, 44-45 (1989): 99-102.
} 
Historias llenas de ficción que han sido útiles para borrar todo vestigio común y así marcar sus espacios y diferencias. Como señala los autores del "Manifiesto":

[...] El futuro público del pasado queda en manos de los historiadores, si estamos dispuestos a mirar por las ventanas de nuestro estudio y pensar en la historia, no como propiedad de un pequeño gremio de colegas profesionales, sino como la herencia legítima de millones [...] Puede que no sea sorprendente, entonces, que tengamos una crisis de gobernanza global, que todos estamos a merced de mercados financieros no regulados, y que el cambio climático antropogénico amenaza nuestra estabilidad política y la supervivencia de las especies. Para poner estos desafíos en perspectiva, y para combatir el corto plazo de nuestro tiempo, necesitamos con urgencia los puntos de vista del gran angular y de largo alcance que solo los historiadores pueden ofrecer. $^{57}$

Esta mirada del gran angular y de largo alcance, pueden encontrar un buen resguardo en los trabajos desarrollados durante los últimos años, respecto a la Historia del presente, la historia global y transancional, que ha venido avanzando en Latinoamérica (al parecer también para otros espacios, como refutó Cohen y Mandler a Guildi y Armitage). ${ }^{58}$ Evidentemente estos nuevos estudios no ocupan todavía el primer lugar en los trabajos historiográficos, prevaleciendo aun las miradas comparadas o nacionales, pero existe un gran potencial en los trabajos publicados durante los últimos años que invitan a pensar la historia más allá de las fronteras. Este es un desafío aún pendiente, pero muy necesario para revitalizar la mirada del historiador en el espacio público y renovar la mirada de larga duración que en su momento planteó Braudel, donde la historia como anecdotario ha alcanzado bastante relevancia desplazando a la mirada compleja del pensamiento histórico sobre los problemas cruciales del presente.

Por último debemos señalar que, compartiendo las conclusiones de Hunt, ${ }^{59}$ las evidencias muestran que en general no han desaparecido las investigaciones de larga duración -como pudimos ver para el caso Latinoamericano- sino que más bien se ha ido generando una tendencia hacia el presentismo en las interpretaciones históricas. En este sentido, el problema no sería el corto plazo o el largo plazo, sino la búsqueda de explicaciones históricas que emerjan de esta miradas, para lo cual varios investigadores en el mundo están trabajando por desarrollar análisis micro-históricos o globales, que sobrepasan la mirada del presente, incluyendo espacios amplios y con temas candentes o controversiales, lo que abre la puerta a un mayor involucramiento de los historiadores en los problemas actuales. $^{60}$

\section{Profile}

\footnotetext{
${ }^{57}$ D. Armitage y J. Guldi, The History Manifesto, 2.

${ }^{58}$ D. Cohen y P. Mandler, "The History Manifesto: A Critique", 532-533.

${ }^{59}$ L. Hunt, "Does History Need a Reset?", 250.

${ }^{60}$ Véase: Ivan Jablonka, Laëtitia o el fin de los hombres (Barcelona: Anagrama, 2017); Yuval Noah, Homo deus (Santiago: Debate, 2014); Brenda E. Stevenson, The Contested Murder of Latasha Harlins: Justice, Gender, and the Origins of the LA Riots (Oxford: Oxford University Press, 2013); Natalie Zemon Davis, Trickster Travels: A Sixteenth-Century Muslim between Worlds (New York: Hill and Wang, 2007).
} 
David Aceituno Silva es Profesor Jornada Completa en el Instituto de Historia de la Pontificia Universidad Católica de Valparaíso (Chile). Magíster en Historia (PUCV) y Doctor en Historia Contemporánea de América Latina (U. Salamanca, España). Becario Postdoctoral de la Fundación Carolina, España y del Coimbra Group. Sus investigaciones se orientan a la Historia del Tiempo Presente, Chile Reciente y Memoria.

David Aceituno Silva is a full-time Professor at the History Institute of the Pontificia Universidad Católica de Valparaíso (Chile). Master in History (PUCV) and Doctor in Contemporary History of Latin America (U. Salamanca, Spain). Postdoctoral Fellow of the Carolina Foundation, Spain and the Coimbra Group. His research is oriented to the History of Present Time, Recent Chile and Memory

Ricardo Iglesias Segura es Profesor Jornada Completa en el Instituto de Historia de la Pontificia Universidad Católica de Valparaíso (Chile). Es Magíster en Historia (PUCV) y Doctor en Historia (U. Valladolid, España). Sus investigaciones se orientan a Historia de Chile, la educación y los nacionalismos.

Ricardo Iglesias Segura is a full-time Professor at the History Institute of the Pontificia Universidad Católica de Valparaíso (Chile). He holds a Master's Degree in History (PUCV) and a $\mathrm{PhD}$ in History (U. Valladolid, Spain). His research focuses on the History of Chile and nationalism.

\section{Bibliografía}

Abdón Mateos, "Historia, Memoria, Tiempo Presente”, Hispania Nova, 1 (1998)

Ana María Stuven y Marco Pamplona (eds.), Estado y Nación en Chile y Brasil en el siglo XIX (Santiago: Ediciones Universidad Católica de Chile, 2009)

Andrew Shryock y Daniel Lord Smail, Deep History: The Architecture of Past and Present (USA: University California Press, 2012)

Ángel Soto, "Historia del presente: estado de la cuestión y conceptualización”, Historia Actual Online, HAOL 3 (2004): 101-116

Antônio A. S. Zuin y Lucídio Bianchetti, "O produtivismo na era do 'publique, apareça ou pereça’: um equilíbrio difícil e necessário”, Cadernos de Pesquisa, vol. 45, 158 (2015): 726750 
Barbara Weinstein, "Pensando la historia más allá de la nación: la historiografía de América Latina y la perspectiva transnacional", Aletheia, vol. 3, 6 (2013)

Brenda E. Stevenson, The Contested Murder of Latasha Harlins: Justice, Gender, and the Origins of the LA Riots (Oxford: Oxford University Press, 2013)

Brian Loveman y Elizabeth Lira, Políticas de reparación. Chile 1990-2004 (Santiago: LOM Ediciones, 2005)

Bruce Mazlish, "Comparing Global History to World History", The Journal of Interdisciplinary, vol. 28, 3 (1998): 385-395

Carlos Aguirre, "El largo siglo XX de la historiografía latinoamericana contemporánea: 1870-¿2025? Puntos de partida para su reconstrucción”, Historia Agenda, Año I. Nueva Época, 3 (2003)

Carlos Aguirre, Ensayos braudelianos: Itinerarios intelectuales y aportes historiográficos de Fernand Braudel (Rosario: Argentina, 2000)

Carlos Aguirre, Fernand Braudel y las Ciencias Humanas (Barcelona: Editorial Montesinos, 1996)

Carlos Guilherme Mota, "Ecos da historiografia francesa no Brasil: Apontamentos e desapontamentos", en Leyla Perrone-Moisés, In Do positivismo à desconstrução: Idéias francesas na América (Sao Paulo: EdUSP, 2003)

Collins Cath, Katherine Hite y Alfredo Joignant, Las políticas de la memoria en Chile: desde Pinochet a Bachelet (Santiago: Ediciones UDP, 2013)

Cristina Moyano, "La historia política en el bicentenario: entre la historia del presente y la historia conceptual. Reflexiones sobre la nueva historia política", Revista de Historia Social y de las Mentalidades, vol. 15, 1 (2011): 227-245

Daniel Camacho y Rafael Menjívar, Los Movimientos populares en América Latina (Chile: Siglo XXI editores, 2005)

Daniel Ovalle, "Pensar la conciencia histórica contemporánea. Historicidad y teoría de la historia”, en Pablo Aravena (ed.), Representación histórica y nueva experiencia del tiempo (Valparaíso: América en movimiento, 2019)

David Aceituno, "Historia del tiempo presente: reflexiones para la investigación en Chile", en David Aceituno y Baldomero Estrada (coords.), Reflexiones y Miradas a nuestro Pasado Reciente. Actas de la I Jornada de Historia de Chile Contemporáneo (Valparaíso: PUCV, 2013) 
David Armitage y Jo Guldi, The History Manifesto (Cambridge: Cambridge University Press, 2014)

David Christian, Maps of Time: An Introduction to Big History (USA: University California Press, 2005)

David M. Ricci, The Tragedy of Political Science. Politics, Scholarship and Democracy (Londres: Yale University Press, 1984)

Deborah Cohen y Peter Mandler, "The History Manifesto: A Critique", American Historical Review, vol. 120, 2 (2015)

Edmundo O’Gorman, La invención de América (México: Fondo de Cultura Económica, 1977)

Eduardo Cavieres y Cristoval Aljovín de Losada (comps.), Chile-Perú. Perú-Chile. 18201920. Desarrollos políticos, económicos y culturales (Valparaíso: Pontificia Universidad Católica de Valparaíso, Convenio Andrés Bello, Universidad Mayor San Marcos, 2005)

Eduardo Cavieres y Fernando Cajías de la Vega (coords.) Chile-Bolivia, Bolivia-Chile: 1820-1930. Desarrollos Políticos, Económicos y Culturales (Valparaíso: Ediciones Universitarias de Valparaíso, 2008)

Elizabeth Jelin (comp.), Las conmemoraciones: las disputas en las fechas 'in-felices' (Madrid: Siglo XXI, 1998)

Emiliano Canto, "Un texto en tres duraciones: Braudel y El Mediterráneo", Temas Antropológicos, Revista Científica de Investigaciones Regionales, vol. 34, 2 (2012): 155-178

Erato Paris, "L'époque brésiliene de Fernand Braudel (1935-1937) et les origines intellectuelles de La Mediterranée et le méditerranéen à l'époque de Philippe II', Storia della Storiografia, 30 (1996): 31-56

Fernand Braudel, El Mediterráneo: el espacio y la historia (México: Fondo de Cultura Económica, 1989)

Fernand Braudel, Escritos sobre la historia (México: Fondo de Cultura Económica, 1991)

Fernand Braudel, La Historia y las Ciencias Sociales (Madrid: Editorial Alianza. S. A., 1970)

Fernando Camacho, "Memorias enfrentadas: las reacciones a los informes nunca más de Argentina y Chile", Persona y sociedad, 67, Vol. XXII, 2 (2008): 67-99 
Fernando García de Cortázar, "El historiador entre el poder y la nada", Cuenta y razón del pensamiento actual, 44-45 (1989): 99-102

Francois Bédarida, "Definición, método y práctica de la Historia del Tiempo Presente", Cuadernos de Historia Contemporánea, 20 (1998)

François Ewald, "El Imperio de una historia", Revista Historias, 13 (1986): 10-15

Horacio Verbitsky y Juan Pablo Bohoslawsky, Cuentas Pendientes. Los cómplices económicos de la dictadura (Buenos Aires: Siglo XXI Editores, 2013)

Ian Merkel, "Fernand Braudel, Brazil, and the Empire of French Social Science Newly Translated Sources from the 1930s", French Historical Studies, vol. 40, 1 (2017): 129-160

Isabel Hofmeyr y Matthew Connelly, "AHR Conversation: On Transnational History", American Historical Review, vol. 111, 5 (2006): 1441-1464

Isabel Torres, La crisis del sistema democrático (Santiago: Ed. Universitaria, 2014)

Ivan Jablonka, Laëtitia o el fin de los hombres (Barcelona: Anagrama, 2017)

João M Paraskeva, Capitalismo académico (Portugal: Pedago, 2009)

Joël Cornette, “Fernand Braudel et l'économie-monde”, L'Histoire, 270 (2002): 51-53

John D French, Magnus Mörner y Julia Fawaz Viñuela, "Comparative Approaches to Latin American History", Latin American Research Review, vol. 17, 2 (1982): 55-89

Jorge Baradit, Historia secreta de Chile (Santiago: Editorial Sudamericana, 2015)

Jorge Baradit, Historia secreta de Chile II (Santiago: Editorial Sudamericana, 2016)

Jorge Baradit, Historia secreta de Chile III (Santiago: Editorial Sudamericana, 2017)

Julián Casanova, "Secretos de Estado" El País, 03 de diciembre del 2010. Recuperado de: http://www.elpais.com/articulo/opinion/Secretos/Estado/elpepuopi/20101203elpepiopi_5/T es [consulta 3 de octubre, 2018]

Louis-Bernard Robitaille, "El Mediterráneo en los tiempos de Braudel", Revista Historias, 13 (1986): 3-10

Luís Corrêa Lima, Fernand Braudel e o Brasil. Vivência e Brasilianismo. 1935-1945 (Brasil: EDUSP, 2010) 
Lynn Hunt, “Does History Need a Reset?”, Annales: Histoire, Sciences Sociales, vol. 70, 2 (2015)

Marc Bloch, "Pour une Histoire Comparée des sociétés européennes”, Mélanges historiques, 1 (1963)

María Ligia Coelho, "América Latina: historia comparada, historias conectadas, historia transnacional", Anuario Facultad de Humanidades y Artes (UNR), 24 (2011)

María Olivia Mönckeberg, El saqueo de los grupos económicos al Estado de Chile (Chile: Ediciones B, 2001)

Martha Herrera y Carol Pertuz, "Educación y políticas de la memoria sobre la historia reciente de América Latina", Revista Colombiana de Educación, 71 (2016): 79-10

Natalie Zemon Davis, Trickster Travels: A Sixteenth-Century Muslim between Worlds (New York: Hill and Wang, 2007)

Nelly Richard, Latencias y sobresaltos de la memoria inconclusa (Chile 1990-2015) (Córdoba: Editorial Villa María, 2018)

Pablo Rubio, Los civiles de Pinochet. La derecha en el régimen militar chileno, 1983-1990 (Santiago: Dibam-Centro de Investigaciones Diego Barros Arana, Santiago, 2013)

Patrice Mcsherry, Los estados depredadores: la Operación Cóndor y la guerra encubierta en América Latina (Chile: LOM, 2009)

Paul Connerton, How Societes Remember (Cambridge: University Press, 1989)

Paulo Iumatti, "Pontos de partida para o estudo do diálogo entre Caio Prado Jr. e Fernand Braudel", en Antonio Gilberto Nogueira y Adelaide Gonçalves (eds.), Caio Prado Jr: Legado de um saber fazer histórico (Sao Paulo: Plebeu Gabinete de Leitura, 2013)

Peter Burke, La revolución historiográfica francesa. La escuela de Annales, 1929-1989 (Barcelona: Gedisa 1993)

Pierre Rosanvallon, "Para una historia conceptual de lo político. Nota de trabajo", Prismas Revista de Historia Intelectual, 6 (2002): 123-136

Reinhart Koselleck, Futuro pasado. Para una semántica de los tiempos histórico (Barcelona: Paidós, 1993)

Reinhart Koselleck, "Einleitung", Geschichtliche Grundbegriffe, XXVII. Citado en Alejandro Cheirif, "La teoría y metodología de la historia conceptual en Reinhart Koselleck", Historiografías, revista de historia y teoría, 7 (2014): 92 
Sanjay Subrahmanyam, "Connected histories: notes towards a reconfiguration of early modern Eurasia", Víctor Lieberman (ed.), Beyond Binary Histories. Re-imagining Eurasia to c. 1830 (Michigan: The University of Michigan Press, 1999)

Serge GruzinskI, O pensamento mestiço (Sao Paulo: Companhia das Letras, 2001)

Serpaj, La complicidad del poder económico con la dictadura: El caso Mercedes Benz. Buenos Aires (Buenos Aires: Servicio Paz y Justicia Equipo, 2003)

Silvio Zavala, Las conquistas de Canarias y América (Canaria: Cabildo Insular de Gran Canaria 1991)

Thomas Piketty, El capital en el siglo XXI (México: Fondo de cultura económica, 2014)

Yuval Noah, Homo deus (Santiago: Debate 2014)

\section{Profile}

David Aceituno Silva y Ricardo Iglesias Segura. Instituto de Historia Pontificia Universidad Católica de Valparaíso (PCVC) (Chile).

David Aceituno Silva es Magister en Historia (PUCV), Doctor en Historia Contemporánea de América Latina por la Universidad de Salamanca (España), becario Postdoctoral de la Fundación Carolina (España) y del Coimbra Group, y profesor del Instituto de Historia de la PCVC (Chile). Sus investigaciones se orientan a la Historia del Tiempo Presente, la historia reciente de Chile y su memoria.

Ricardo Iglesias Segura es Doctor en Historia por la Universidad de Valladolid (España) y profesor en el Instituto de Historia de la PUCV (Chile). Sus investigaciones se concentran en la historia de Chile y de los nacionalismos.

David Aceituno Silva has a Master in History at the PUCV, a PhD in Contemporary History of Latin America at the University of Salamanca (Spain), a post-graduate fellowship from the Carolina Foundation (Spain) and from the Coimbra Group; and he is also delivering classes at the Institute of History in the PUCV (Chile). His research hinges on the History of the Present, the Chilean recent history and her memory.

Ricardo Iglesias Segura has a PhD in History at the University of Valladolid (Spain), and he is also delivering classes at the Institute of History PUCV (Chile), his research focusing upon the history of Chile and of nationalisms. 
Fecha de recepción: 30 de octubre de 2018.

Fecha de aceptación: 17 de junio de 2019.

Publicación: 30 de junio de 2019.

Para citar este artículo: David Aceituno y Ricardo Iglesias, "Pensar la historia del presente en Latinoamérica: reflexiones a partir del 'History Manifiesto of Cambridge", Historiografías, 17 (enero-junio, 2019): pp. 4-26. 